August 9, 2021

\title{
Singular factorizations, self-adjoint extensions, and applications to quantum many-body physics
}

\author{
Edwin Langmann*, Ari Laptev** and Cornelius Paufler*** \\ * Mathematical Physics, KTH Physics, AlbaNova, SE-106 91 Stockholm, Sweden \\ ** Department of Mathematics, KTH, SE-100 44 Stockholm, Sweden \\ *** Theoretische Elementarteilchen-Physik, Institut für Physik, \\ Johannes-Gutenberg-Universität, D-55099 Mainz, Germany
}

\begin{abstract}
We study self-adjoint operators defined by factorizing second order differential operators in first order ones. We discuss examples where such factorizations introduce singular interactions into simple quantum mechanical models like the harmonic oscillator or the free particle on the circle. The generalization of these examples to the many-body case yields quantum models of distinguishable and interacting particles in one dimensions which can be solved explicitly and by simple means. Our considerations lead us to a simple method to construct exactly solvable quantum many-body systems of Calogero-Sutherland type.
\end{abstract}

\section{Introduction.}

In this paper we define and study peculiar self-adjoint extensions of simple differential operators like the one defining the quantum mechanical harmonic oscillator. We also find the explicit solution of some of the resulting quantum mechanical models and discuss their physical interpretations. These examples provide amusing illustrations that the mathematical theory of Schrödinger operators [K, RS1, RS2, BS], and in particular the distinction between formally hermitian and self-adjoint operators, can have important physical consequences even in elementary quantum mechanics. Moreover, these examples allow a straightforward generalization to the case of an arbitrary number of particles, and the resulting many-body models describe strongly interacting systems which can be solved by elementary means. These models include, as special cases, the ones studied by Girardeau [G], which have received considerable interest recently since it has become possible to realize and study them experimentally $[\mathrm{P}$. We stress that these models can be solved even in the general case of distinguishable particles, and that they can be studied by much simpler methods than other well-known integrable quantum many-body systems LL, Y, C2, IS. Moreover, our considerations lead us to a remarkably simple and general approach to exactly solvable quantum many-body systems of Calogero-Sutherland type C2, S, OP, which seems new. 
The starting point for this work was a paradox for the differential operator $-\partial_{x}^{2}+x^{2}$ defining quantum harmonic oscillator, $-\infty<x<\infty$ and $\partial_{x}:=\partial / \partial x$. The paradox arises if one confronts the formal factorization

$$
-\partial_{x}^{2}+x^{2}=\left(\partial_{x}-x+\frac{1}{x}\right)\left(-\partial_{x}-x+\frac{1}{x}\right)+3 \equiv Q^{*} Q+3
$$

and $Q^{*} Q \geq 0$, with the well-known fact that the groundstate eigenvalue of the harmonic oscillator Hamiltonian $H=-\partial_{x}^{2}+x^{2}$ is 1 . It is interesting to note that a variant of this paradox was already discussed by Calogero in 1969; see [C1, second paragraph after Eq. (3.24).

The paradox above is only one of many, and they are resolved by observing that each such factorization gives rise to a different quantum mechanical Hamiltonian defined by the Friedrichs extension. These examples have in common that a simple differential operator with an obvious factorization also allows for alternative factorizations where singularities are introduced, and this implies a restriction on the domain of functions on which the resulting self-adjoint operator is defined. This restriction has a natural physical interpretation as strong repulsive interaction.

We will present several examples leading to interesting quantum mechanical model with additional interactions and which are exactly solvable in the sense that all eigenfunctions and eigenvalues can be found explicitly and by simple means. ${ }^{1}$ In particular, for each non-negative integer $n$ we will find a quantum mechanical Hamiltonian $H_{n}$ which is defined by the differential operator of the harmonic oscillator differential operator $-\partial_{x}^{2}+x^{2}$ but is bounded from below by $2 n+1$. Moreover, in addition to the standard harmonic oscillator $H=H_{0}$ also the Hamiltonian $H_{1}$ defined by the factorization in Eq. (II) is exactly solvable, while the other $H_{n \geq 2}$ are only partially solvable, i.e., only a finite number of eigenstates and eigenvalues can be found exactly. Two other examples are provided by the differential operator $-\partial_{x}^{2}-\gamma(\gamma+1) \cosh ^{-2}(x)$ often referred to as Pöschl-Teller potential, and the differential operator $-\partial_{x}^{2}$ for $-\pi<x<\pi$ describing a free particle on the circle. In the latter example we will show that, for every $\alpha>0$, the factorization

$$
-\partial_{x}^{2}=\left(\partial_{x}+\alpha \cot (\alpha x)\right)\left(-\partial_{x}+\alpha \cot (\alpha x)\right)+\alpha^{2}
$$

defines a unique self-adjoint operator $H_{\alpha}$ bounded from below by $\alpha^{2}$. Moreover, this Hamiltonian is exactly solvable if and only if $\alpha$ is integer or half integer.

While we believe that these examples are of pedagogical interest, our first main result is that much of what we say can be naturally extended to the many-body case. This yields many-body systems describing distinguishable particles moving in one dimension and interacting through a strong local two-body interactions, and also these models can be solved exactly and by simple means.

Our considerations described above lead us, somewhat unexpectedly, to our second main result which is on Calogero-Sutherland type systems: As we will see, all our one-

\footnotetext{
${ }^{1}$ To illustrate what we mean by this we recall that the spectrum of the Hamiltonian defined by the differential operator $-\partial_{x}^{2}$ on all $C^{\infty}$-functions on an interval and general boundary conditions is given by a transcendental equation in general which can be solved only numerically. These examples are not exactly solvable in this sense.
} 
particle examples mentioned above are related to Schrödinger operators $H=-\partial_{x}^{2}+V(x)$ which have exact eigenfunctions of the form

$$
\psi_{n}(x)=P_{n}(\eta(x)) \mathrm{e}^{-w(x)}, \quad n=0,1,2, \ldots, N_{\max }
$$

where $P_{n}$ is a polynomial of order $n, \eta$ and $w$ are real-valued $C^{2}$ functions, and $N_{\text {max }} \leq \infty$. These quantum mechanical models are exactly solvable in the sense that these eigenfunctions $\psi_{n}$ and the corresponding eigenvalues $E_{n}$ can be found explicitly. While we will restrict our discussions to our three examples: harmonic oscillator, Pöschl-Teller potential, and free particle on the circle, it is important to note that there are many more: in Table 1 we list the well-known examples associated with the classical orthogonal polynomials, but even this list is not complete. Our second main result is a surprisingly simple argument showing that, to any such model, the corresponding quantum-many body given by the Schrödinger operator

$$
H_{N}=\sum_{j=1}^{N}\left(-\partial_{x_{j}}^{2}+V\left(x_{j}\right)\right)+\lambda(\lambda-1) \sum_{1 \leq j<k \leq N} W\left(x_{j}, x_{k}\right)
$$

and the following particular two-body interaction

$$
W(x, y)=\frac{\eta^{\prime}(x)^{2}+\eta^{\prime}(y)^{2}}{(\eta(x)-\eta(y))^{2}}+\frac{2 \eta^{\prime}(x) w^{\prime}(x)-\eta^{\prime \prime}(x)-2 \eta^{\prime}(y) w^{\prime}(y)+\eta^{\prime \prime}(y)}{\eta(x)-\eta(y)}-W_{0}
$$

defines an exactly solvable quantum many-body system; $\eta^{\prime}(x):=\partial_{x} \eta(x)$, and $W_{0}$ is an arbitrary constant which can be fixed to some convenient value. More specifically, this arguments leads to a simple proof of the following:

Proposition: Let $H=-\partial_{x}^{2}+V(x)$ be a self-adjoint Schrödinger operator on $L^{2}([a, b])$, $-\infty \leq a<b \leq \infty$, with exact eigenfunctions as specified in Eq. (2) ff. Then the corresponding many-body Schrödinger operator $H_{N}$ in Eqs. (3)-(4) defines a quantum-many body systems with the following exact ground state,

$$
\Psi_{0}\left(x_{1}, \ldots, x_{N}\right)=\mathrm{e}^{-\sum_{j=1}^{N} w\left(x_{j}\right)} \prod_{1 \leq j<k \leq N}\left(\eta\left(x_{j}\right)-\eta\left(x_{k}\right)\right)^{\lambda},
$$

provided $N<N_{\max }+1$ and $\lambda>0$ is such that $\Psi_{0}$ is square-integrable. The corresponding eigenvalue is determined by the one-particle eigenvalues $E_{n}$ as follows,

$$
\mathcal{E}_{0}=\lambda^{2} \sum_{n=0}^{N-1}\left(E_{n}-E_{0}\right)+N E_{0}-\frac{1}{2} \lambda(\lambda-1) N(N-1) W_{0}
$$

(A concise proof can be found in the last paragraph of Section 6.)

In fact, we found that it is possible compute all other bound states and corresponding eigenvalues for all these systems exactly and in a unified manner [HL. As we will discuss 


\begin{tabular}{c|cccc} 
& Name & $\psi_{n}(x)$ & $V(x)$ & $E_{n}$ \\
\hline I & Jacobi & $\left(\sin \frac{x}{2}\right)^{\alpha}\left(\cos \frac{x}{2}\right)^{\beta} P_{n}^{(\alpha, \beta)}(\cos x)$ & $\frac{4 \alpha^{2}-1}{16 \sin ^{2} \frac{x}{2}}+\frac{4 \beta^{2}-1}{16 \cos ^{2} \frac{x}{2}}$ & $\left(n+\frac{\alpha+\beta+1}{2}\right)^{2}$ \\
II & Gegenbauer & $(\sin x)^{\alpha} C_{n}^{(\alpha)}(\cos x)$ & $\frac{\alpha(\alpha-1)}{\sin ^{2} x}$ & $(n+\alpha)^{2}$ \\
III & Chebyshev & $T_{n}(\cos x)$ & 0 & $n^{2}$ \\
IV & Laguerre & $\mathrm{e}^{-x^{2} / 2} x^{(\alpha+1) / 2} L_{n}^{(\alpha)}\left(x^{2}\right)$ & $4 n+2 \alpha+2$ & $x^{2}+\frac{4 \alpha^{2}-1}{4 x^{2}}$
\end{tabular}

Table 1: Solutions of the Schrödinger equation $\left(-\partial_{x}^{2}+V(x)-E_{n}\right) \psi_{n}(x)=0$ associated with classical orthogonal polynomials; adapted from Table 22.6 in [AS].

in Section 7, special cases of this result yield various well-known exactly solvable systems of Calogero-Sutherland type $[\mathrm{C} 2,[\mathrm{~S}, \mathrm{OP}, \mathrm{BF}$, but there are other interesting examples which we have not seen discussed in the physics literature.

The plan of this paper is as follows. In the next section we resolve in detail the paradox described above using the theory of Friedrichs extensions. This provides a general recipe for finding other such examples. In Section 3 we present our one-body examples, and in Section 4 we discuss their physical interpretation. In Section 5 we discuss the extension of our results to the many-body case. Our method to find quantum-many body systems with exact groundstates is explained in Section 6. We end with a few remarks in Section 7.

\section{Factorizations and selfadjoint extensions.}

We first recall some standard facts from the operator theory in Hilbert spaces. Let $A_{0}$ be a symmetric operator in a separable Hilbert space $\mathcal{H}$ with scalar product $(\cdot, \cdot)$ (linear in the second argument) and norm $\|\cdot\|$, and let $\mathcal{D}\left(A_{0}\right)$ be the domain of the operator $A_{0}$ which is a dense subset in $\mathcal{H}$. We assume that $A_{0}$ is bounded from below, i.e., there exists a constant $E_{0} \in \mathbb{R}$ such that

$$
\left(A_{0} u, u\right) \geq E_{0}\|u\|^{2}, \quad u \in \mathcal{D}\left(A_{0}\right) .
$$

The sesquilinear form $a_{0}[u, v]=\left(A_{0} u, v\right)$ defined for $u, v \in d\left[a_{0}\right]=\mathcal{D}\left(A_{0}\right)$ can be completed with respect to the norm defined by

$$
a_{0}[u, u]+\left(E_{0}+1\right)\|u\|^{2} .
$$

Such a completion is denoted by $a$ and its domain by $d[a]$. By standard arguments (see for example $[\mathrm{BS}]$ ) there is a unique selfadjoint operator $A$ corresponding to the closed form $a$ whose domain $\mathcal{D}(A) \subset d[a]$. This operator is called the Friedrichs extension of the operator $A_{0}$.

Let $V$ be a real function defined on open subset $\Omega \subseteq \mathbb{R}^{N}$, and let $H$ be a self-adjoint Schrödinger operator in $L^{2}(\Omega)$ with some boundary conditions.

$$
H u(x)=-\Delta u(x)+V(x) u(x) .
$$

This operators generates a quadratic form $h[u, u]$ defined on functions from $d[h]$. We assume that $H$ has discrete spectrum $\left\{E_{n}\right\}_{n=0}^{K}$, with the corresponding orthonormal in 
$L^{2}(\Omega)$ system of eigenfunctions $\left\{u_{n}\right\}_{n=0}^{K}$ where $K \leq \infty$ (i.e. $H$ may also have continuous spectrum). It can be easily checked directly that the differential expression $-\Delta+V(x)$ can formally be factorized as

$$
-\Delta+V=Q_{n}^{*} Q_{n}+E_{n}
$$

where

$$
Q_{n}=-\nabla_{x}+\frac{\nabla u_{n}}{u_{n}}
$$

It is well-known that the ground state $u_{0}$ can be chosen strictly positive and the factorization (9) does not give any problem. However, considering higher eigenfunctions we obtain singularities in the right hand side of (10) due to the zero set $S$ of $u_{n}, S=\left\{x: u_{n}(x)=0\right\}$. In order to define the selfadjoint operator corresponding to the operator $Q^{*} Q$ we begin with the quadratic form

$$
h_{n}[u, u]=\int_{\Omega}\left|-\nabla_{x} u+\frac{\nabla u_{n}}{u_{n}} u\right|^{2} \mathrm{~d}^{N} x
$$

whose domain $d\left[h_{n}\right]$ consists of functions $u \in d[h]$ such that $u(x)=0$ for $x \in S$.

The corresponding Friedrichs extension gives us a selfadjoint operator which we denote by $H_{n}$. Although

$$
H_{n}=Q_{n}^{*} Q_{n}+E_{n}=-\Delta+V
$$

coincides with $H=-\Delta+V$ defined by (8) regarding its action on functions as a differential expression, its domain is very different and consists of functions not only just satisfying the boundary conditions of the original operator $H$ but being equal to zero on $S$. This automatically makes the domain $d\left[h_{n}\right]$ smaller compared with the domain of the original operator $H$ and "lifts up" the spectrum.

In particular, this answers the question concerning the formal inequality which follows from (111), that is

$$
H=Q_{n}^{*} Q_{n}+E_{n} \geq E_{n} .
$$

The equality in the right hand side of this latter equation is wrong because the domains

of the operator $H$ and $Q_{n}^{*} Q_{n}$ are different and therefore $H \neq Q_{n}^{*} Q_{n}+E_{n}$ as Hilbert space operators.

\section{Some examples.}

Here we shall give some simple examples where the above construction can be worked out more explicitly.

3.a. Harmonic oscillator.

Let $H$ be the operator of the harmonic oscillator on $\mathbb{R}$,

$$
H=-\partial_{x}^{2}+x^{2}
$$

The eigenvalues and eigenfunctions of this operator are well-known and equal to $E_{n}=$ $2 n+1, n=0,1, \ldots$, and

$$
\psi_{n}(x)=\mathcal{H}_{n}(x) e^{-x^{2} / 2},
$$


where $\mathcal{H}_{n}$ are Hermite polynomials,

$$
\mathcal{H}_{n}(x)=\left(2^{n} n !\right)^{-1 / 2} \pi^{-1 / 4}(-1)^{n} e^{x^{2}} \partial_{x}^{n} e^{-x^{2}} .
$$

As in (11) the operators $H_{n}$ can be defined via

$$
H_{n}=Q_{n}^{*} Q_{n}+E_{n}=\left(\partial_{x}+\frac{\psi_{n}^{\prime}}{\psi_{n}}\right)\left(-\partial_{x}+\frac{\psi_{n}^{\prime}}{\psi_{n}}\right)+E_{n} .
$$

According to Section 2 the latter expression coincides with $-\partial_{x}^{2}+x^{2}$ as a differential expression but not as a selfadjoint operator. In particular, when $n=0$,

$$
\frac{\psi_{0}^{\prime}}{\psi_{0}}=-x
$$

and we obtain the standard factorization of the harmonic oscillator

$$
H_{0}=-\partial_{x}^{2}+x^{2}=\left(\partial_{x}-x\right)\left(-\partial_{x}-x\right)+1
$$

If $n=1$ then

$$
\frac{\psi_{1}^{\prime}}{\psi_{1}}=-x+\frac{1}{x}
$$

and we obtain the factorization

$$
H_{1}=-\partial_{x}^{2}+x^{2}=\left(\partial_{x}-x+\frac{1}{x}\right)\left(-\partial_{x}-x+\frac{1}{x}\right)+3
$$

It is interesting to note that not only $H_{0}$ but also $H_{1}$ defines an exactly solvable system in the sense that all its eigenfunctions can be computed explicitly: Using that the eigenfunctions $\psi_{n}(x)$ of $H_{0}$ in Eq. (13) $f f$. are even and odd for even and odd integers $n$, respectively, we conclude that the eigenfunctions $u_{n}(x)$ of $H_{1}$ are given by

$$
u_{2 n+1}(x)=\psi_{2 n+1}(x), \quad u_{2 n}(x)=\psi_{2 n+1}(x) \operatorname{sign}(x), \quad n=0,1,2 \ldots
$$

with the usual sign function $\operatorname{sign}(x)$ : the odd eigenfunctions of $H_{1}$ are the same as for the standard harmonic oscillator, but the even ones are very different. The spectrum of $H_{1}$ thus has multiplicity two, $E_{2 n}=E_{2 n+1}=4 n+3$.

The other exotic harmonic oscillator Hamiltonians $H_{n \geq 2}$ are only partially solvable: as discussed in Section 4 below, we can determine the $(n+1)$-fold degenerate ground state of $H_{n \geq 2}$ explicitly, but it is not possible to compute its excited states analytically.

3.b. Pöschl-Teller potential.

Let

$$
H=-\partial_{x}^{2}-\frac{\gamma(\gamma+1)}{\cosh ^{2} x}
$$

for $\gamma>0$. It is well-known that this operator has $[\gamma](=$ largest integer smaller or equal to $\gamma$ ) square integrable eigenfunctions $\psi_{n}, n=0,1, \ldots,[\gamma]-1$, with corresponding eigenvalues

$$
E_{n}=-(\gamma-n)^{2}
$$


These eigenfunctions are given by

$$
\psi_{n}(x)=\cosh ^{-\gamma}(x) \sum_{0 \leq s \leq n / 2}(-1)^{s} \frac{n !(\gamma-n) !}{4^{s}(n-2 s) !(\gamma+s-n) ! s !} \sinh ^{n-2 s}(x)
$$

with $r !:=\Gamma(r+1)$ for non-integers $r$, as can be checked by straightforward computations.

In particular, the lowest eigenvalue of this operator is $E_{0}=-\gamma^{2}$ and the corresponding eigenfunction is $\psi_{0}(x)=\cosh ^{-\gamma}(x)$. Then

$$
\frac{\psi_{0}^{\prime}}{\psi_{0}}=-\gamma \tanh (x)
$$

and therefore one can obtain the following factorization

$$
H_{0}=-\partial_{x}^{2}-\frac{\gamma(\gamma+1)}{\cosh ^{2} x}=\left(\partial_{x}-\gamma \tanh (x)\right)\left(-\partial_{x}-\gamma \tanh (x)\right)-\gamma^{2}
$$

defining the standard selfadjoint operator $H_{0}=H$ given by the differential operator $-\partial_{x}^{2}+\gamma(\gamma+1) \cosh ^{-2}(x)$. The second eigenvalue is $E_{1}=-(\gamma-1)^{2}$ and the corresponding eigenfunction is given by $\psi_{1}(x)=\cosh ^{-\gamma}(x) \sinh (x)$, for $\gamma>1$. Then

$$
\frac{\psi_{1}^{\prime}}{\psi_{1}}=\operatorname{coth}(x)-\gamma \tanh (x)
$$

which implies that

$$
\begin{aligned}
H_{1}=-\partial_{x}^{2}-\frac{\gamma(\gamma+1)}{\cosh ^{2} x}= & \left(\partial_{x}+\operatorname{coth}(x)-\gamma \tanh (x)\right) \\
& \times\left(-\partial_{x}+\operatorname{coth}(x)-\gamma \tanh (x)\right)-(\gamma-1)^{2}
\end{aligned}
$$

which defines another selfadjoint operator $H_{1}$, and similarly for $H_{n \geq 2}$. Again it is possible to compute all eigenfunctions and eigenvalues of $H_{1}$ in terms of the ones of $H_{0}$, similarly as in the harmonic oscillator case.

3.c. Free particle on the circle.

This example is slightly more subtle. For arbitrary $\alpha>0$ we define a self-adjoint operator on $L^{2}([-\pi, \pi])$ by the factorization

$$
H_{\alpha}=-\partial_{x}^{2}=\left(\partial_{x}+\alpha \cot (\alpha x)\right)\left(-\partial_{x}+\alpha \cot (\alpha x)\right)+\alpha^{2}
$$

which is obtained with the odd eigenfunctions $\sin (\alpha x)$ of $-\partial_{x}^{2}$. The subtle point now concerns the correct boundary conditions at $x= \pm \pi$. According to our general theory in Section 2, we need to start with the self-adjoint operator $H=-\partial_{x}^{2}$ with boundary conditions such that $\sin (\alpha x)$ is indeed its eigenfunction, and it is not difficult to see that these conditions are as follows,

$$
u^{\prime}(-\pi)=u^{\prime}(\pi) \text { and } u(\pi)-u(-\pi)=-2 \tan (\alpha \pi) u^{\prime}(\pi)
$$


for functions $u$ in the domain of $H$. Thus only for integers $\alpha$ this corresponds to periodic boundary conditions, and only in this case can we interpret $H$ as Hamiltonian of a particle on a circle. We conclude that the eigenfunctions of $H_{\alpha}$ are defined by the same boundary conditions at $x= \pm \pi$ together with the requirements that they vanish at all points $x$ where $\sin (\alpha x)=0$.

If we interpret $H_{\alpha}$ as Hamiltonian of a particle moving on a circle, then we not only have infinitely strong repulsive interactions at the points $x$ where $\sin (\alpha x)$ vanishes but, in addition, a singular interaction at the point $x= \pm \pi$ whose strength depends on $\alpha$ so that it vanishes at integer values of $\alpha$ and becomes infinitely strong if $\alpha$ is half integer.

In particular, if $\alpha=1 / 2$, then the operator

$$
H_{1 / 2}=-\partial_{x}^{2}=\left(\partial_{x}+\frac{1}{2} \cot (x / 2)\right)\left(-\partial_{x}+\frac{1}{2} \cot (x / 2)\right)+\frac{1}{4}
$$

is defined on functions $u$ satisfying a Dirichlet boundary condition at zero (from the singularity of cot at zero) and Neumann boundary conditions at $-\pi$ and $\pi$,

$$
u(0)=u^{\prime}(-\pi)=u^{\prime}(\pi)=0
$$

Our discussion above implies that, for integers and half-integers $\alpha$, one can determine all eigenfunctions and eigenvalues of $H_{\alpha}$ explicitly and by elementary computations, but otherwise part of the spectrum is determined by a transcendental equation: the eigenfunctions are determined by the conditions $u(k \pi / \alpha)=0$ for integer $k$ and the boundary conditions in Eq. (18), and the latter are non-trivial unless $2 \alpha$ is an integer.

For example, for $\alpha=1$ these eigenfunctions are given by

$$
u_{ \pm, n}(x)=\Theta( \pm x) \sin (n x)
$$

with $n=1,2, \ldots$. The corresponding eigenvalues are $n^{2}$ and two fold-degenerate. For $\alpha=1 / 2$ the explicit eigenfunctions are

$$
u_{ \pm, n}=\Theta( \pm x) \sin ((n+1 / 2) x / 2) \quad n=0,1,2, \ldots
$$

with corresponding two-fold degenerate eigenvalues $(n+1 / 2)^{2}$.

We finally mention that it is amusing to visualize the $\alpha$-dependence of the singular interactions and the spectrum of the Hamiltonian $H_{\alpha}$.

\section{Physical Interpretation.}

We first give a physics argument providing an intuitive resolution of the paradox mentioned after Eq. (1).

The paradox arises due to the singularity in the factorization in Eq. (11) at $x=0$. One can regularize this singularity and consider the following family of Hamiltonians,

$$
H^{(\varepsilon)}=\left(\partial_{x}-x+\frac{x}{x^{2}+\varepsilon^{2}}\right)\left(-\partial_{x}-x+\frac{x}{x^{2}+\varepsilon^{2}}\right)+3,
$$


with a regularization parameter $\varepsilon>0$. By a straightforward computation one finds

$$
H^{(\varepsilon)}=-\partial_{x}^{2}+x^{2}+\frac{\varepsilon^{2}\left(1+2 \varepsilon^{2}+2 x^{2}\right)}{\left(x^{2}+\varepsilon^{2}\right)^{2}} .
$$

Even though the second term vanishes for $\varepsilon=0$, it is obviously relevant in the limit $\varepsilon \downarrow 0$ : Recalling that $\delta_{\varepsilon}(x)=\varepsilon /\left(x^{2}+\varepsilon^{2}\right) \pi$ is a sequence of $C^{\infty}$ functions converging to the delta distribution as $\varepsilon \downarrow 0$, we see that this term gives an additional singular interaction of infinite strength at $x=0$ : formally, the second term converges to $\lim _{\mathcal{E} \downarrow 0}\left(\pi \delta_{\varepsilon}(x)\right)^{2}=\pi^{2} \delta(0) \delta(x)=\lim _{g \rightarrow+\infty} g \delta(x)$. This interaction forces the eigenfunctions of the Hamiltonian $\lim _{\varepsilon \downarrow 0} H^{(\varepsilon)}$ to vanish at $x=0$. It is plausible that this latter Hamiltonian is identical with the one defined by the factorization in (11), and this is indeed the case, as discussed below.

As shown in the previous section, the eigenfunctions of Hamiltonians $H=-\partial_{x}^{2}+V(x)$ on $L^{2}([a, b]),-\infty \leq a<b \leq \infty$, defined by the factorization

$$
H_{n}=-\partial_{x}^{2}+V(x)=\left(\partial_{x}+u_{n}^{\prime}(x) / u_{n}(x)\right)\left(-\partial_{x}+u_{n}^{\prime}(x) / u_{n}(x)\right)+E_{n}
$$

can be fully characterized by the (simple) zeros $x_{n, j}$ of the eigenfunction $u_{n}$ of $-\partial_{x}+V(x)$ : $u_{n}\left(x_{n, j}\right)=0$ for $j=1,2, \ldots, n$ implies that $u\left(x_{n, j}\right)=0$ for all eigenfunctions $u$ of $H_{n}$. One thus can interpret $H_{n}$ as the strong coupling limit $g \rightarrow \infty$ limit of the following Hamiltonian,

$$
H_{n}^{(g)}=-\partial_{x}^{2}+V(x)+\sum_{j=1}^{n} g \delta\left(x-x_{n, j}\right) .
$$

Indeed, the eigenfunctions $u$ of this latter Hamiltonian are defined by $-u^{\prime \prime}+V u=E u$ for $x \neq x_{n, j}$, together with the boundary conditions

$$
u\left(x_{n, j}+0\right)=u\left(x_{n, j}-0\right), \quad u^{\prime}\left(x_{n, j}+0\right)-u^{\prime}\left(x_{n, j}-0\right)=g u\left(x_{n, j}+0\right),
$$

and for $g \rightarrow \infty$ the latter reduce to $u\left(x_{n, j}\right)=0$. Thus the factorization corresponds to adding infinitely strong repelling delta-potentials at the locations of the zeros of the eigenfunction $u_{n}$. This implies that the ground state of $H_{n}$ is $n+1$-fold degenerate: all states $u_{0, j}(x)$ which are equal to $u_{n}(x)$ in an interval between two adjacent zeros $x_{n, j}$ and zero otherwise are eigenfunctions of $H_{n}$ with eigenvalue $E_{n}$. However, the excited states cannot be found in such a simple manner in general.

\section{Exactly solvable many-body systems in $1 \mathrm{D}$.}

We now show that the examples above allow a straightforward extension to the $N$-particle case, and this gives rise to exactly solvable interacting many body systems of distinguishable particles.

5.a. Interacting particles in a harmonic oscillator potential.

Our first example is for the operator

$$
H_{N}=\sum_{j=1}^{N}\left(-\partial_{x_{j}}^{2}+x_{j}^{2}\right)
$$


on $\mathbb{R}^{N}$. This self-adjoint operator is defined by the standard factorization

$$
H_{N}=\sum_{j=1}^{N}\left(-\partial_{x_{j}}^{2}+x_{j}^{2}\right)=\sum_{j=1}^{N}\left(\partial_{x_{j}}-x_{j}\right)\left(-\partial_{x_{j}}-x_{j}\right)+N
$$

and it describes an arbitrary number, $N$, of noninteracting, identical particles moving in an external harmonic oscillator potential. The eigenfunctions of $H_{N}$ obviously are given by products of the one particle eigenfunctions defined in Section 3.a,

$$
\psi_{\boldsymbol{n}}(\boldsymbol{x})=\prod_{j=1}^{N} \psi_{n_{j}}\left(x_{j}\right), \quad n_{j}=0,1,2, \ldots
$$

where we use the shorthand notation $\boldsymbol{x}=\left(x_{1}, \ldots, x_{N}\right)$ and similarly for $\boldsymbol{n}$, and the eigenvalue is

$$
\mathcal{E}_{\boldsymbol{n}}=\sum_{j=1}^{N}\left(2 n_{j}+1\right)
$$

Using the huge degeneracy of these eigenfunctions we can construct eigenfunctions of $H_{N}$ which vanish at all coinciding points $x_{j}=x_{k}$ by antisymmetrization,

$$
f_{\boldsymbol{n}}(\boldsymbol{x})=\sum_{P \in S_{N}}(-1)^{|P|} \prod_{j=1}^{N} \psi_{n_{j}}\left(x_{P j}\right), \quad 0 \leq n_{1}<n_{2}<\ldots<n_{N}<\infty .
$$

These are exactly the fermion eigenfunctions of $H_{N}$, of course: exchanging any two particles gives a minus sign. The fermion wave function with the lowest eigenvalue is

$$
f_{0}(\boldsymbol{x})=\text { const } \operatorname{det}_{1 \leq j, k \leq N}\left(\psi_{j-1}\left(x_{k}\right)\right)
$$

where const is some irrelevant non-zero constant which we will later fix to some convenient value. To compute $f_{0}$ we use the explicit form of the harmonic oscillator eigenfunctions in Eq. (13),

$$
f_{\boldsymbol{n}_{0}}(\boldsymbol{x})=\mathrm{e}^{-\sum_{j} x_{j}^{2} / 2} \operatorname{det}_{1 \leq j, k \leq N}\left(\mathcal{H}_{j-1}\left(x_{k}\right)\right) .
$$

Since the Hermite polynomials are of the form $\mathcal{H}_{n}(x)=c_{n} x^{n}+\ldots$ with dots some linear combinations of $\mathcal{H}_{m<n}$ and $c_{n}$ non-zero constants, we can replace in this determinant $\mathcal{H}_{j-1}\left(x_{k}\right)$ by $c_{j-1} x_{k}^{j-1}$, and a well-known identity of the Vandermonde determinant implies that

$$
f_{0}(\boldsymbol{x})=\mathrm{e}^{-\sum_{j=1}^{N} x_{j}^{2} / 2} \prod_{1 \leq j<k \leq N}\left(x_{j}-x_{k}\right)
$$

is the fermion ground state of the operator $H_{N}$ with the eigenvalue $\sum_{j=0}^{N-1}(2 j+1)=N^{2}$ (we fixed const so as to absorb factors of $c_{j}$ and a possible sign). Then obviously

$$
\frac{1}{f_{0}(\boldsymbol{x})} \frac{\partial}{\partial x_{j}} f_{0}(\boldsymbol{x})=-x_{j}+\sum_{\substack{k=1 \\ k \neq j}}^{N} \frac{1}{x_{j}-x_{k}}
$$


We thus conclude that the differential operator defining $H_{N}$ also can be factorized as follows,

$$
H_{N, 1}=\sum_{j=1}^{N}\left(-\partial_{x_{j}}^{2}+x_{j}^{2}\right)=\sum_{j=1}^{N} Q_{j}^{*} Q_{j}+N^{2}, \quad Q_{j}=-\partial_{x_{j}}-x_{j}+\sum_{\substack{k=1 \\ k \neq j}}^{N} \frac{1}{x_{j}-x_{k}}
$$

and this defines another self-adjoint operator.

We now discuss how to determine all eigenfunctions $f(\boldsymbol{x})$ of $H_{N, 1}$; see $\mathbf{G}$ for a similar discussion. As in Section 2 we conclude that the eigenfunction have to vanish at coinciding points,

$$
f\left(x_{1}, x_{2}, \ldots, x_{N}\right)=0 \quad \text { if } x_{j}=x_{k} \text { for some } j \neq k, j, k=1,2, \ldots, N,
$$

and thus all fermion eigenfunctions $f_{\boldsymbol{n}}$ of $H_{N}$ in Eq. (21) are also eigenfunctions of $H_{N, 1}$ with the same eigenvalues. It is not difficult to see that all these are $C^{\infty}$ eigenfunctions of $H_{N, 1}$, but they obviously do not span the full Hilbert space $L^{2}\left(\mathbb{R}^{N}\right)$ but only its fermion subspace. To find the missing eigenfunctions we divide the space $\mathbb{R}^{N}$ in fundamental domains which can be parameterized by permutations $P \in S_{N}$,

$$
\Delta_{P}:=\left\{\boldsymbol{x}=\left(x_{1}, \ldots, x_{N}\right) \in \mathbb{R}^{N} \mid-\infty<x_{P 1}<x_{P 2}<\ldots<x_{P N}<\infty\right\} .
$$

There are $N$ ! distinct such domains, and the closure of the union of all these domains equals to full space, $\overline{\cup_{P \in S_{N}} \Delta_{P}}=\mathbb{R}^{N}$. Moreover, the boundaries of these domains correspond to the hyperplanes $x_{j}=x_{k}$. We thus need to find all functions which are $C^{\infty}$ and obey the eigenvalue equation for $H_{N}$ in the interior of all these domains $\Delta_{P}$ and vanish at all their boundaries. The functions in Eq. (21) obey both these conditions, but since the derivatives of eigenfunctions need not be continuous at coinciding point also the functions

$$
f_{\boldsymbol{n}}^{P}(\boldsymbol{x})=\left\{\begin{array}{cc}
f_{\boldsymbol{n}}(\boldsymbol{x}) & \text { for } \boldsymbol{x} \in \Delta_{P} \\
0 & \text { otherwise }
\end{array}, \quad 0 \leq n_{1}<n_{2}<\ldots<n_{N}<\infty, \quad P \in S_{N}\right.
$$

are legitimate eigenfunctions: they obey the correct eigenvalue equation with the eigenvalue in Eq. (20) at non-coinciding points, and they also obey all boundary conditions in Eq. (24). It is not difficult to verify that these eigenfunctions $f_{\boldsymbol{n}}(\boldsymbol{x})$ span the full Hilbert space $L^{2}\left(\mathbb{R}^{N}\right)$.

Similarly as discussed in Section 4 , we can interpret $H_{N, 1}$ as the strong coupling limit, $g \rightarrow \infty$, of the following Hamiltonian

$$
H_{N}^{(g)}=\sum_{j=1}^{N}\left(-\partial_{x_{j}}^{2}+x_{j}^{2}\right)+g \sum_{1 \leq j<k \leq N} \delta\left(x_{j}-x_{k}\right)
$$

describing $N$ particles moving on the real line in the harmonic oscillator potentials and interacting with two-body delta function potentials. This corresponds to the fact that the quadratic form of the operator $H_{N, 1}$ is split into $N$ ! independent problems in $L^{2}\left(\Delta_{P}\right)$ with Dirichlet boundary conditions. 
5.b. Interacting particles in a Pöschl-Teller potential.

We now consider the operator

$$
H_{N}=\sum_{j=1}^{N}\left(-\partial_{x_{j}}^{2}-\gamma(\gamma+1) \frac{1}{\cosh ^{2}\left(x_{j}\right)}\right)
$$

describing $N$ noninteracting particles in a weakly confining $\cosh ^{-2}$-potential. For $\gamma>N$ the fermion ground state of this Hamiltonian is $f_{0}$ as in (22) but now with the eigenfunctions $\psi_{n}$ in (17), and by an argument as in Section 5.a we conclude that

$$
f_{0}(\boldsymbol{x})=\prod_{j=1}^{N} \cosh ^{-\gamma}\left(x_{j}\right) \prod_{1 \leq j<k \leq N}\left(\sinh \left(x_{j}\right)-\sinh \left(x_{k}\right)\right)
$$

with corresponding eigenvalue

$$
\mathcal{E}_{0}=-\sum_{n=0}^{N-1}(\gamma-n)^{2}=-\frac{N}{6}(2 N-1)(N-1)+\gamma N(N-1)-N \gamma^{2} .
$$

We thus can define the following Hamiltonian different from $H_{N}$,

$$
H_{N, 1}=\sum_{j=1}^{N} Q_{j}^{*} Q_{j}+\mathcal{E}_{0}
$$

with

$$
Q_{j}=-\partial_{x_{j}}+\frac{1}{f_{0}} \partial_{x_{j}} f_{0}=-\partial_{x_{j}}-\gamma \tanh \left(x_{j}\right)+\sum_{\substack{k=1 \\ k \neq j}}^{N} \frac{\cosh \left(x_{j}\right)}{\sinh \left(x_{j}\right)-\sin \left(x_{k}\right)} .
$$

As in Section 5.a we can interpret $H_{N, 1}$ as the strong coupling limit of the Hamiltonian $H_{N}$ in (27) with two body delta interactions enforcing that the eigenfunctions vanish at coinciding points $x_{j}=x_{k}$, and all eigenvalues and eigenfunctions of this interacting many-body Hamiltonian can be determined explicitly from the fermion wave functions and corresponding eigenvalues of the non-interacting Hamiltonian $H_{N}$. The example here is, however, quite interesting from a physical point of view since, in addition to manybody bound states, there are also scattering states and, in general, one has mixed states where only some of the particles are trapped and the others are free.

5.c. Interacting particles on the circle.

Let

$$
H_{N}=-\Delta=-\sum_{j=1}^{N} \partial_{x_{j}}^{2}
$$

on $N$ dimensional torus $\mathbb{T}^{N}$. The right hand side of (30) can be considered as a trivial factorization of the periodic Laplace operator. We note that

$$
\psi_{n}(x)=\left(\mathrm{e}^{\mathrm{i} x}\right)^{n} \mathrm{e}^{-\mathrm{i}(N-1) x / 2}, \quad n=0, \pm 1, \pm 2, \ldots
$$


all are eigenfunctions of the corresponding one-particle Hamiltonian $-\partial_{x}^{2}$ with eigenvalue $\left(n-\frac{1}{2}(N-1)\right)^{2}$, and thus $f_{0}$ as defined in (22) is an fermion eigenstate of the operator (301) on $\mathbb{T}^{N}$ with the eigenvalue $\sum_{n=0}^{N-1}\left(n-\frac{1}{2}(N-1)\right)^{2}=\frac{1}{12} N\left(N^{2}-1\right)$. By straightforward computations we find that

$$
f_{0}(\boldsymbol{x})=\prod_{1 \leq j<k \leq N} \sin \frac{1}{2}\left(x_{j}-x_{k}\right)
$$

and therefore, by analogy with (23) , we can consider the factorization

$$
H_{N, 1}=-\sum_{j=1}^{N} \partial_{x_{j}}^{2}=\sum_{j=1}^{N} Q_{j}^{*} Q_{j}+\frac{1}{12} N\left(N^{2}-1\right)
$$

where

$$
Q_{j}=-\partial_{x_{j}}-\frac{1}{f_{0}} \partial_{x_{j}} f_{0}=-\partial_{x_{j}}+\sum_{\substack{k=1 \\ k \neq j}}^{N} \frac{1}{2} \cot \frac{1}{2}\left(x_{j}-x_{k}\right) .
$$

Note that the singularities enforce that eigenfunctions of $H_{N, 1}$ vanish at points $x_{j}=x_{k}$ modulo $2 \pi$, and thus we can interpret $H_{N, 1}$ as describing particles moving on a circle of length $2 \pi,-\pi \leq x_{j} \leq \pi$, interacting with a strong two-body delta interaction.

As in the previous examples this factorization splits the operator $H_{N, 1}$ into an orthogonal sum of operators in fundamental domains parameterized by permutations $P \in S_{N}$, and the eigenfunctions of $H_{N, 1}$ can all be determined explicitly as antisymmetrized products of the eigenfunctions of the corresponding non-interacting Hamiltonian $H_{N}$.

\section{A method to find Calogero-Sutherland type systems.}

As already mentioned, our considerations in the previous section are closely related to arguments which are well-known in the context of Calogero-Sutherland type systems [C1]. In this sections we explore this relation in more detail. Through that we are naturally lead to a surprisingly simple approach to a large class of Calogero-Sutherland type systems.

One well-known method to find quantum integrable systems is to write down a many-body state of Jastrow form

$$
\Psi_{0}(\boldsymbol{x})=\prod_{j=1}^{N} \mathrm{e}^{-w\left(x_{j}\right)} \prod_{1 \leq j<k \leq N} g\left(x_{j}, x_{k}\right)^{\lambda}, \quad \lambda \geq 0,
$$

for some $C^{2}$ functions $w$ and $g$, and then construct a many-particle Hamiltonian

$$
H_{N}=\sum_{j=1}^{N} \mathcal{Q}_{j}^{*} \mathcal{Q}_{j}-\mathcal{E}_{0}, \quad \mathcal{Q}_{j}=-\partial_{x_{j}}+\frac{1}{\Psi_{0}} \partial_{x_{j}} \Psi_{0}
$$

with $\mathcal{E}_{0}$ some real, conveniently chosen constant. Then $H_{N}$ obviously defines self-adjoint operator $\geq \mathcal{E}_{0}$, and $\mathcal{Q}_{j} \Psi_{0}=0 \forall j$ implies that $\Psi_{0}$ is an exact groundstate of $H_{N}$ with eigenvalue $\mathcal{E}_{0}$. In general, such a Hamiltonian $H_{N}$ describes a system of particles with two- 
and three-body interactions, and only for very particular functions $w$ and $g$ the three-body terms cancel. These cases are of particular interest since they lead to physically interesting Hamiltonians of the form as in Eq. (3) which, in many cases, are exactly solvable. Famous such examples are $w(x)=\frac{1}{2} x^{2}, g(x, y)=(x-y)$ and $w(x)=0, g(x, y)=\sin \frac{1}{2}(x-y)$ corresponding to the models discovered by Calogero [C2]:

$$
H_{N}=\sum_{j=1}^{N}\left(-\partial_{x_{j}}^{2}+x_{j}^{2}\right)+\lambda(\lambda-1) \sum_{1 \leq j<k \leq N} \frac{2}{(x-y)^{2}}
$$

and Sutherland [S]:

$$
H_{N}=-\sum_{j=1}^{N} \partial_{x_{j}}^{2}+\lambda(\lambda-1) \sum_{1 \leq j<k \leq N} \frac{1}{2 \sin ^{2} \frac{1}{2}\left(x_{j}-x_{j}\right)},
$$

respectively. Olshanetsky and Perelomov found interesting variants of the CalogeroSutherland models related to root systems; see [OP] and references therein.

Obviously, the Calogero- and Sutherland models are very closely related to our examples discussed in Sections 5.a and 5.c, respectively. It is interesting to note that our considerations there provide an alternative proof of the key identity leading to these models. Indeed, if we check the identity in (23) by a direct computation, we find that it is equivalent to the well-known functional identity which cancels the three-body terms in the Calogero model:

$$
\frac{1}{\left(x_{j}-x_{k}\right)\left(x_{j}-x_{\ell}\right)}+c y c l=0
$$

for $j, k, \ell$ all different, where ' $+c y c l$ ' is short for adding the two terms of the same form obtained by cyclic permutations of the indices, $(j, k, \ell) \rightarrow(k, \ell, j)$ and $(j, k, \ell) \rightarrow(\ell, j, k)$. However, since we have a direct proof of (23) based on a basic identity of the Vandermonde determinant, we found an alternative proof of this functional identity. Similarly, the identity in Eqs. (31)-(32) is equivalent to the well-known functional identity

$$
\cot \left(\frac{1}{2}\left(x_{j}-x_{k}\right)\right) \cot \frac{1}{2}\left(x_{j}-x_{\ell}\right)+c y c l=-1,
$$

used to cancel the three-body terms in the Sutherland model.

It therefore is natural to ask: is there a Calogero-Sutherland type system corresponding to our example in Section 5.b? We now can use a similar method as above to find such a system: we can use Eqs. (28)-(29) to derive the functional identity

$$
\frac{\cosh ^{2}\left(x_{j}\right)}{\left(\sinh \left(x_{j}\right)-\sinh \left(x_{k}\right)\right)\left(\sinh \left(x_{j}\right)-\sinh \left(x_{\ell}\right)\right)}+c y c l=1,
$$

which then can be used to cancel three-body terms and prove that

$$
\Psi_{0}(\boldsymbol{x})=\prod_{j=1}^{N} \cosh ^{-\gamma}\left(x_{j}\right) \prod_{1 \leq j<k \leq N}\left(\sinh \left(x_{j}\right)-\sinh \left(x_{k}\right)\right)^{\lambda}
$$


is the exact ground state of the Hamiltonian

$$
H_{N}=\sum_{j=1}^{N}\left(-\partial_{x_{j}}^{2}-\frac{\gamma(\gamma+1)}{\cosh ^{2}\left(x_{j}\right)}\right)+\lambda(\lambda-1) \sum_{1 \leq j<k \leq N} \frac{\cosh ^{2}\left(x_{j}\right)+\cosh ^{2}\left(x_{k}\right)}{\left(\sinh \left(x_{j}\right)-\sinh \left(x_{k}\right)\right)^{2}}
$$

Moreover, it is straightforward to show that all other bound states and corresponding eigenvalues can be found using Sutherland's method $[\mathrm{S}] .^{2}$

It is interesting to note that our argument can be easily extended to a rather large class of examples: assume a one-particle Hamiltonian $H=-\partial_{x}^{2}+V(x)$ which has exact eigenstates of the form

$$
\psi_{n}(x)=P_{n}(\eta(x)) \mathrm{e}^{-w(x)}, \quad n=0,1,2, \ldots
$$

with eigenvalue $E_{n}$, where $P_{n}$ is a polynomial of order $n$ and $w$ and $\eta$ are $C^{2}$ functions. Then $f_{0}$ in (22) is obviously an eigenstate of the many-body Hamiltonian

$$
\sum_{j}\left(-\partial_{x_{j}}^{2}+V\left(x_{j}\right)\right)
$$

with eigenvalue $\mathcal{E}_{0}=\sum_{j=0}^{N-1} E_{n}$. As in Section 5 we can conclude that

$$
f_{0}(\boldsymbol{x})=\prod_{j=1}^{N} \mathrm{e}^{-w\left(x_{j}\right)} \prod_{1 \leq j<k \leq N}\left(\eta\left(x_{j}\right)-\eta\left(x_{k}\right)\right),
$$

and using this formula for $f_{0}$ the identity

$$
\sum_{j=1}^{N}\left(-\partial_{x_{j}}^{2}+V\left(x_{j}\right)\right)=\sum_{j=1} Q_{j}^{*} Q_{j}+\mathcal{E}_{0}, \quad Q_{j}=-\partial_{x_{j}}+\frac{1}{f_{0}} \partial_{x_{j}} f_{0}
$$

implies a functional identity expressing three-body terms through two-body terms. We now compute

$$
\mathcal{H}=\sum_{j=1} \mathcal{Q}_{j}^{*} \mathcal{Q}_{j}, \quad \mathcal{Q}_{j}=-\partial_{x_{j}}+\frac{1}{\Psi_{0}} \partial_{x_{j}} \Psi_{0}
$$

with $\Psi_{0}$ in (5) , and using this functional identity we can cancel all three-body terms and thus find that $\mathcal{H}=H_{N}-\mathcal{E}_{0}$ with $H_{N}$ in (3)-(44) and $\mathcal{E}_{0}$ in (6). Since obviously $\mathcal{H} \Psi_{0}=0$ and $\mathcal{H} \geq 0$, we obtain the result stated in the Proposition in Section 1. Table 1 gives other interesting examples of this result. We plan to give a comprehensive discussion of all these models and their solution in a future publication $\mathrm{HL}$.

\section{Final comments.}

We should mention that the model in (34) is closely related to the well-known $C_{N}$-variant of the Sutherland model defined by

$$
H_{C_{N}}=\sum_{j=1}^{N}\left(-\partial_{x_{j}}^{2}+\frac{\gamma(\gamma+1)}{\sinh ^{2}\left(x_{j}\right)}\right)+\lambda(\lambda-1) \sum_{1 \leq j<k \leq N}\left(\frac{1}{2 \sin ^{2} \frac{1}{2}\left(x_{j}-x_{j}\right)}+\right.
$$

\footnotetext{
${ }^{2}$ E. Langmann, unpublished.
} 
see $\mathrm{OP}$ and references therein. This Hamiltonian describes identical particles in an external, repulsive potential and with repulsive two-body interactions, and it only has scattering states (purely continuous spectrum). One can show that by shifting $x_{j} \rightarrow$ $x_{j}+\mathrm{i} \pi / 2$, this Hamiltonian is converted to the one in (34), up to an additive constant. However, these shifts changes the physics of the model dramatically: the Hamiltonian in (34) describes interacting particles in a weakly confining potential, and it thus has, in addition to bound states and scattering states, also mixed states where some of the particles are confined and others free. To our knowledge, this situation has not been studied in the context of Calogero-Sutherland type systems. We therefore think it would be interesting to study this model in detail which, however, is beyond the scope of the present paper.

There are other special cases which seem less known in the physics literature even though, to our opinion, they also of interest in physics. The examples listed in Table 1 lead to systems which were previously known in the mathematics literature; see [BF, [DX] and references therein. We should mention that these latter systems are closely related to the $B C_{N}$ variants of the Calogero- (IV) and Sutherland model (I-III) OP, respectively. However, our result nevertheless seems useful since it is the starting point of an alternative, systematic and unified approach to these systems and their solutions, including examples which are hardly known [HL].

Acknowledgement We would like to thank Göran Lindblad for providing with us a helpful tool for numerical experiments [L. We are grateful for helpful discussions with F. Calogero, P. Forrester, M. Hallnäs, G. Lindblad and J. Mickelsson. C.P. was supported by Deutsche Forschungsgemeinschaft under the Emmy-Noether program. E.L. was supported by the Swedish Science Research Council (VR). E.L. and A.L. are supported by the Göran Gustafsson Foundation and the European grant "ENIGMA".

\section{References}

[AGHH] S. Albeverio, F. Gesztesy, R. Hoegh-Krohn, H. Holden: Solvable models in quantum mechanics, Springer, New York, 1988.

[AS] A. Abramowitz and I. A. Stegun: Handbook of mathematical functions, Dover Publications, Inc., New York, 1972.

[BF] T. H. Baker and P. J. Forrester: The Calogero-Sutherland model and generalized classical polynomials, Commun. Math. Phys. 188 (1997) 175.

[BS] M. Birman, M. Solomyak : Spectral theory of selfadjoint operators in Hilbert space. Mathematics and its Applications (Soviet Series). D. Reidel Publishing Co., Dordrecht, 1987.

[C1] F. Calogero: Solution of a three-body problem in one dimension, J. Math. Phys. 10 (1969) 2191. 
[C2] F. Calogero: Solution of the one-dimensional N body problems with quadratic and/or inversely quadratic pair potentials, J. Math. Phys. 12 (1971) 419.

[DX] C.F. Dunkl and Y. Xu: Orthogonal polynomials of several variables. Cambridge Univ. Press, Cambridge, 2001.

[G] M. Girardeau: Relationship between systems of impenetrable bosons and fermions in one dimensions, J. Math. Phys. 1 (1960) 516.

[K] T. Kato: Perturbation theory for linear operators. Die Grundlehren der mathematischen Wissenschaften in Einzeldarstellungen 132, Springer-Verlag, Berlin, 1966.

[HL] M. Hallnäs and E. Langmann, work in progress.

[L] G. Lindblad, "Quantum Mechanics with MATLAB", available on internet, http://mathphys.physics.kth.se/schrodinger.html.

[LL] E. H. Lieb and W. Liniger, Exact analysis of an interacting bose gas. 1. The general solution and the ground state, Phys. Rev. 130 (1963) 1605.

[OP] M. A. Olshanetsky and A. M. Perelomov, Quantum integrable systems related to Lie algebras, Phys. Rept. 94 (1983) 313.

[P] B. Peredes et.al., Tonks-Girardeau gas of ultracold atoms in optical lattice, Nature 429 (2004) 277.

[RS1] M. Reed, B. Simon : Methods of Modern Mathematical Physics. I: Functional Analysis. Academic Press, New York, 1972.

[RS2] M. Reed, B. Simon : Methods of Modern Mathematical Physics. II: Fourier Analysis, Self-Adjointness. Academic Press, New York, 1975.

[S] B. Sutherland, Exact results for a quantum many body problem in one-dimension, Phys. Rev. A 4 (1971) 2019 and ibid. 5 (1972) 1372.

[Y] C. N. Yang, Some exact results for the many body problems in one dimension with repulsive delta function interaction, Phys. Rev. Lett. 19 (1967) 1312. 